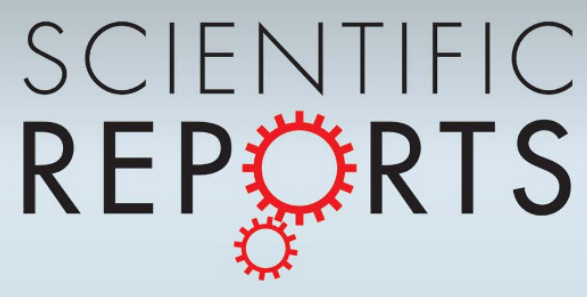

OPEN

SUBJECT AREAS:

MATERIALS PHYSICS

MAGNETIC MATERIALS AND

DEVICES

ELECTRONIC MATERIALS AND

DEVICES

SENSORS

Received

26 August 2011

Accepted

14 December 2011

Published

13 January 2012

Correspondence and requests for materials should be addressed to D.W. (wangdh@nju. edu.cn)

\section{Electric control of magnetism at room temperature}

\author{
Liaoyu Wang, Dunhui Wang, Qingqi Cao, Yuanxia Zheng, Haicheng Xuan, Jinlong Gao \& Youwei Du
}

National Laboratory of Solid State Microstructures, Department of Physics, Nanjing University, Nanjing 210093, People's Republic of China.

In the single-phase multiferroics, the coupling between electric polarization $(\mathrm{P})$ and magnetization $(\mathrm{M})$ would enable the magnetoelectric (ME) effect, namely $M$ induced and modulated by $E$, and conversely $P$ by $\mathrm{H}$. Especially, the manipulation of magnetization by an electric field at room-temperature is of great importance in technological applications, such as new information storage technology, four-state logic device, magnetoelectric sensors, low-power magnetoelectric device and so on. Furthermore, it can reduce power consumption and realize device miniaturization, which is very useful for the practical applications. In an M-type hexaferrite $\mathrm{SrCo}_{2} \mathrm{Ti}_{2} \mathrm{Fe}_{8} \mathrm{O}_{19}$, large magnetization and electric polarization were observed simultaneously at room-temperature. Moreover, large effect of electric field-controlled magnetization was observed even without magnetic bias field. These results illuminate a promising potential to apply in magnetoelectric devices at room temperature and imply plentiful physics behind them.

- he magnetoelectric coupling between the magnetic and electric orders in multiferroics brings about novel physical mechanism and offers the opportunity for attractive applications, such as non-volatile memory technology, four-state logic device and magnetoelectric sensors ${ }^{1-7}$. Of particular interest is electric control of magnetism, which has a promising potential for reducing power consumption and realizing device miniaturization in spintronic devices ${ }^{8,9}$. However, such applications have been limited by weak magnetoelectric effects and low operating temperature in most discovered single-phase multiferroics. Very recently, giant magnetoelectric effects have been reported in some hexaferrites, in which the electric polarization $(P)$ can be induced by their complex magnetic orders ${ }^{10-14}$.

Hexaferrites, which have long been utilized in permanent magnets and microwave devices, can be classified into different types such as $\mathrm{M}, \mathrm{W}, \mathrm{X}, \mathrm{Y}, \mathrm{U}$, and $\mathrm{Z}^{15}$. Their crystal structures can be described as stacked sequences of the basic blocks: $S, R$, and $\mathrm{T}^{15}$. Recently, some significant results about the magnetically induced ferroelectricity and resultant magnetoelectric effects were observed in some Y-, Z- and M-type hexaferrites due to the modifications of spiral magnetic structures by an external magnetic field ${ }^{10-14}$. This phenomena can be understood by the spin current or the inverse Dzyaloshinskii-Moriya models described by the equation

$$
P=A \bar{e}_{i j} \times\left(\bar{S}_{i} \times \bar{S}_{j}\right)
$$

( $\bar{e}_{i j}$ denotes spins at neighboring sites $\mathrm{i}$ and $\mathrm{j}, \bar{e}_{i j}$ denotes the propagation vector of a spiral structure, $A$ denotes the coupling coefficient determined by the exchange interaction and the spin-orbit interaction.), in which the interaction between noncollinear spins can produce the local $P$ through the spin-orbit interaction ${ }^{16-18}$.

Recently, Z-type hexaferrite $\mathrm{Sr}_{3} \mathrm{Co}_{2} \mathrm{Fe}_{24} \mathrm{O}_{41}$ was reported to show an excellent room-temperature magnetoelectric property at low magnetic field $(H)$, which would shed light on the practical applications of magnetoelectric multiferroics ${ }^{13}$. Since the electric resistivity in most of these hexaferrites is not high enough to support an electric field, the manipulation of magnetization by an electric field has rarely been reported, especially at room temperature ${ }^{8}$. Here, we report the discovery of electric control of magnetism in an M-type hexaferrite, $\mathrm{SrCo}_{2} \mathrm{Ti}_{2} \mathrm{Fe}_{8} \mathrm{O}_{19}$ (SCTFO), at room temperature. Furthermore, without magnetic bias field, large effect of electric field-controlled magnetization is observed at room temperature, which would revolutionize the field of lowpower magnetoelectronic devices.

\section{Results}

The M-type hexaferrites, $\mathrm{AFe}_{12} \mathrm{O}_{19}(\mathrm{~A}=\mathrm{Ba}, \mathrm{Sr}, \mathrm{Pb}$, etc.), crystallize in the magnetoplumbite-type structure (the space group $\mathrm{P}_{3} / \mathrm{mmc}$ ) with $\mathrm{Fe}^{3+}$ ions in octahedral $(12 k, 4 f$ and $2 a)$, tetrahedral $(4 f)$ and bipyramidal $(2 b)$ sites, respectively. This lattice structure could be symbolically described as an alternate stacking of 4 building blocks, 
namely $S, S^{*}, R$, and $R^{*}$, which is schematically shown elsewhere ${ }^{13,15}$. The large $\mathrm{A}^{2+}$ ion, which causes a slight perturbation in the lattice around itself, is responsible for the large magnetic uniaxial anisotropy in these hexaferrites, where the crystallographic c-axis is the magnetic easy axis ${ }^{15}$. The substituted M-type hexaferrites have been extensively investigated for the application point of view ${ }^{15,19}$. Among them, the substitution of $\mathrm{Fe}^{3+}$ cations with $\mathrm{Co}^{2+}-\mathrm{Ti}^{4+}$ pair is reported to alter the magnetocrystalline anisotropy and give rise to the noncollinear magnetic structures ${ }^{20,21}$. In this case, $\mathrm{Co}^{2+}$ cations prefer to occupy the octahedrally-coordinated $4 f$ and $12 k$ Wyckoff positions and the magnetic anisotropy changes gradually from an axial anisotropy (along the hexagonal $c$ axis) to a planar anisotropy ( $a, b$ plane of the hexagonal cell) with increasing concentration of $\mathrm{Co}^{2+15,21}$. On the other hand, nonmagnetic $\mathrm{Ti}^{4+}$ cations substituted on the $12 k$ would interrupt the magnetic interactions in a way that the two blocks RS and $\mathrm{R}^{*} \mathrm{~S}^{*}$ become more or less magnetically decoupled ${ }^{20}$. As a result, an intermediate conical magnetic structure was obtained in the Mtype hexaferrite with critical Co-Ti concentration, which can be verified by neutron diffraction and Mössbauer spectroscopy ${ }^{20,22}$.

Figure 1 shows the room-temperature powder X-ray diffraction pattern and Rietveld refinement of polycrystalline SCTFO. All peaks can be indexed as the magnetoplumbite-type cell in the space group $P 6_{3} / m m c\left(a=5.881(1) \AA, b=5.881(1) \AA, c=23.102(8) \AA, \alpha=90.00^{\circ}\right.$, $\left.\beta=90.00^{\circ}, \gamma=120.00^{\circ}\right)$. Table I summarizes the fractional coordinates and occupation factors.

The magnetic hysteresis loops for SCTFO was measured at $300 \mathrm{~K}$. As shown in Fig. 2(a), the sample is ferromagnetic at room temperature with the magnetization of about $30 \mathrm{emu} / \mathrm{g}$ with $H=15 \mathrm{kOe}$. Comparing with $\mathrm{SrFe}_{12} \mathrm{O}_{19}$, the coercivity of SCTFO decrease remarkably (see in the inset of Fig. 2(a)), which is resulted from the reduction in anisotropy field with the substitution of $\mathrm{Co}^{2+}-\mathrm{Ti}^{4+}$ for $\mathrm{Fe}^{3+19}$. The temperature dependence of magnetization $(M-T)$ from $100-800 \mathrm{~K}$ for SCTFO is shown in Fig. 2(b). The Curie temperature of $740 \mathrm{~K}$ for this ferrite is less than that of $\mathrm{SrFe}_{12} \mathrm{O}_{19}{ }^{23}$, which would be ascribed to the substitution of $\mathrm{Fe}^{3+}$ with $\mathrm{Co}^{2+}-\mathrm{Ti}^{4+}$ pair $^{19}$. Based on the results of neutron diffraction and magnetic measurements, the magnetization anomaly at around $420 \mathrm{~K}$ is likely to correspond to the transition from collinear ferrimagnetic phase to the phase with a conical magnetic structure ${ }^{20,22,24}$.

Figure 3(a) shows the temperature dependence of $P$ at zero magnetic field, which is obtained from integrating the magnetoelectric current by time with $400 \mathrm{~K}$ as the initial point. As we know, the reported Y- or Z-type multiferroics hexaferrites, such as $\mathrm{Ba}_{2} \mathrm{Mg}_{2}$ $\mathrm{Fe}_{12} \mathrm{O}_{22}$ or $\mathrm{Sr}_{3} \mathrm{Co}_{2} \mathrm{Fe}_{24} \mathrm{O}_{41}$, show no spontaneous $P$ at zero magnetic field. When an external magnetic field is applied, a transverse conical

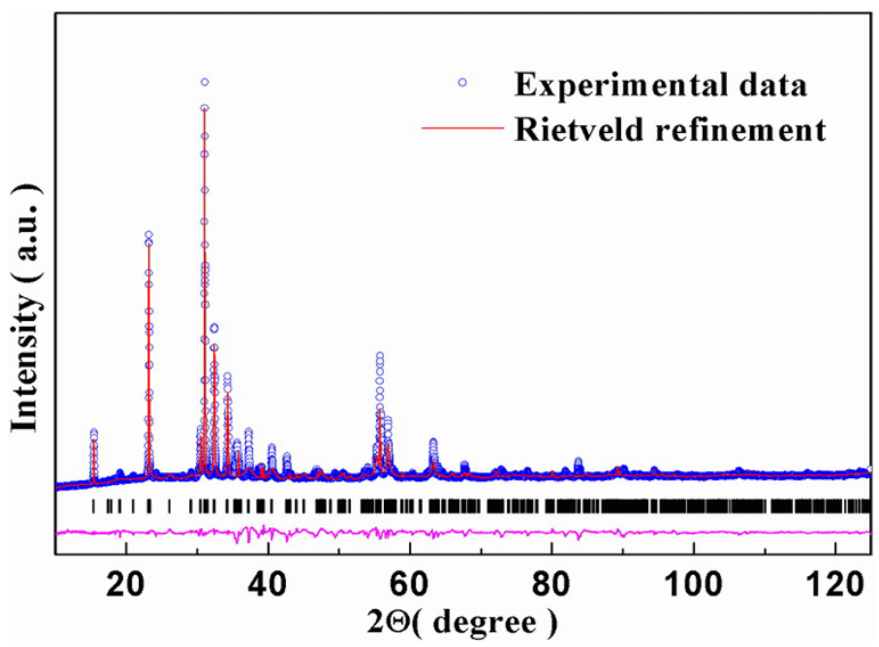

Figure 1 The room-temperature powder X-ray diffraction pattern and Rietveld refinement for polycrystalline SCTFO.
Table I| The fractional coordinates and occupation factors for polycrystalline SCTFO.

\begin{tabular}{llllll} 
Atom & Site & \multicolumn{1}{c}{$x$} & \multicolumn{1}{c}{$y$} & \multicolumn{1}{c}{$z$} & \multicolumn{1}{c}{ SOF } \\
\hline Sr1 & $2 \mathrm{~d}$ & 0.3333 & 0.6667 & 0.25 & 1.0 \\
Fe1 & $2 \mathrm{a}$ & 0.0 & 0.0 & 0.0 & 1.0 \\
Fe2 & $2 \mathrm{~b}$ & 0.0 & 0.0 & $0.6231(16)$ & 0.5 \\
Fe3 & $4 \mathrm{f}$ & 0.6667 & 0.3333 & $0.02714(28)$ & 1.0 \\
Co1 & $4 \mathrm{f}$ & 0.6667 & 0.3333 & $0.2310(8)$ & 1.0 \\
Fe5 & $12 \mathrm{k}$ & $0.1845(4)$ & $0.3691(8)$ & $0.11117(12)$ & 0.66667 \\
Ti1 & $12 \mathrm{k}$ & $0.0973(5)$ & $0.1946(11)$ & $0.11266(25)$ & 0.33333 \\
$\mathbf{O 1}$ & $4 \mathrm{e}$ & 0.0 & 0.0 & $0.1225(17)$ & 1.0 \\
$\mathbf{O 2}$ & $4 \mathrm{f}$ & 0.3333 & 0.6667 & $0.0935(6)$ & 1.0 \\
$\mathbf{O 3}$ & $6 \mathrm{~h}$ & $0.8707(6)$ & $0.7413(13)$ & 0.25 & 1.0 \\
$\mathbf{O 4}$ & $12 \mathrm{k}$ & $0.8462(9)$ & $0.6924(18)$ & $0.03472(20)$ & 1.0 \\
$\mathbf{O 5}$ & $12 \mathrm{k}$ & $0.5387(6)$ & $1.0774(13)$ & $0.11144(24)$ & 1.0 \\
\hline
\end{tabular}

spin structure is induced, which could produce $P$ through the spin current and the inverse Dzyaloshinskii-Moriya mechanism ${ }^{11,13,18}$. However, in the case of SCTFO, it shows spontaneous $P$ at zero magnetic field, indicating that its spin structure has a transverse cycloidal component, which should be further investigated by neutron diffraction measurements. In addition, a sudden decrease of $P$ almost coincides with the anomaly in the $M-T$ curve, suggesting the coupling between the ferromagnetism and ferroelectricity ${ }^{13,14}$. Figure 3(b) shows the $H$ dependence of $P$ for the sample at $300 \mathrm{~K}$. With increasing $H$, the $P$ is considerably suppressed, demonstrating a strong magnetoelectric coupling in SCTFO. As shown in Fig. 2(b), $P$ does not vanish even $H=16 \mathrm{~T}$, indicating that the conical spin structure is robust. Accordingly, there is no anomaly in $M-H$ curve (Figure 3(b)), which is consistent with the behavior of $P$.
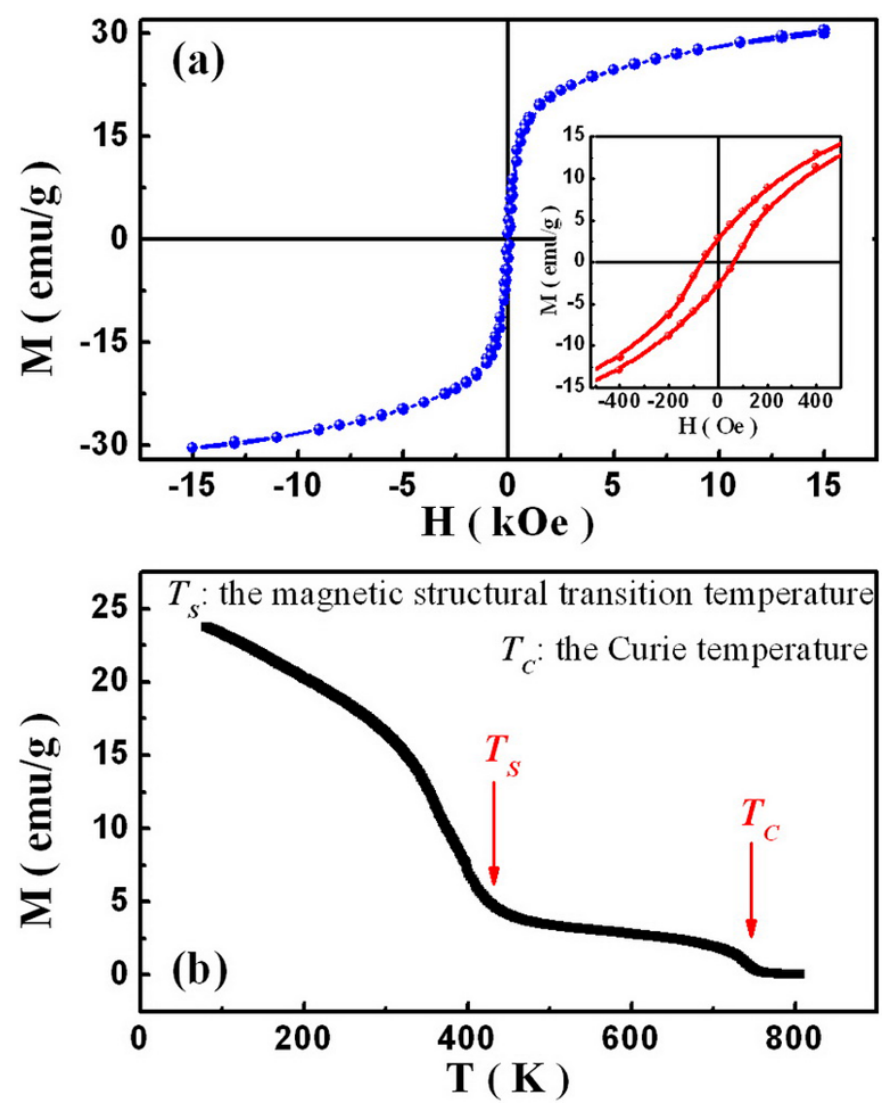

Figure $2 \mid$ (a) The magnetic hysteresis loop for SCTFO at $300 \mathrm{~K}$; the inset of (a) shows the enlarged magnetic hysteresis loop; (b) The variation of $M$ for the sample as a function of $T$. 

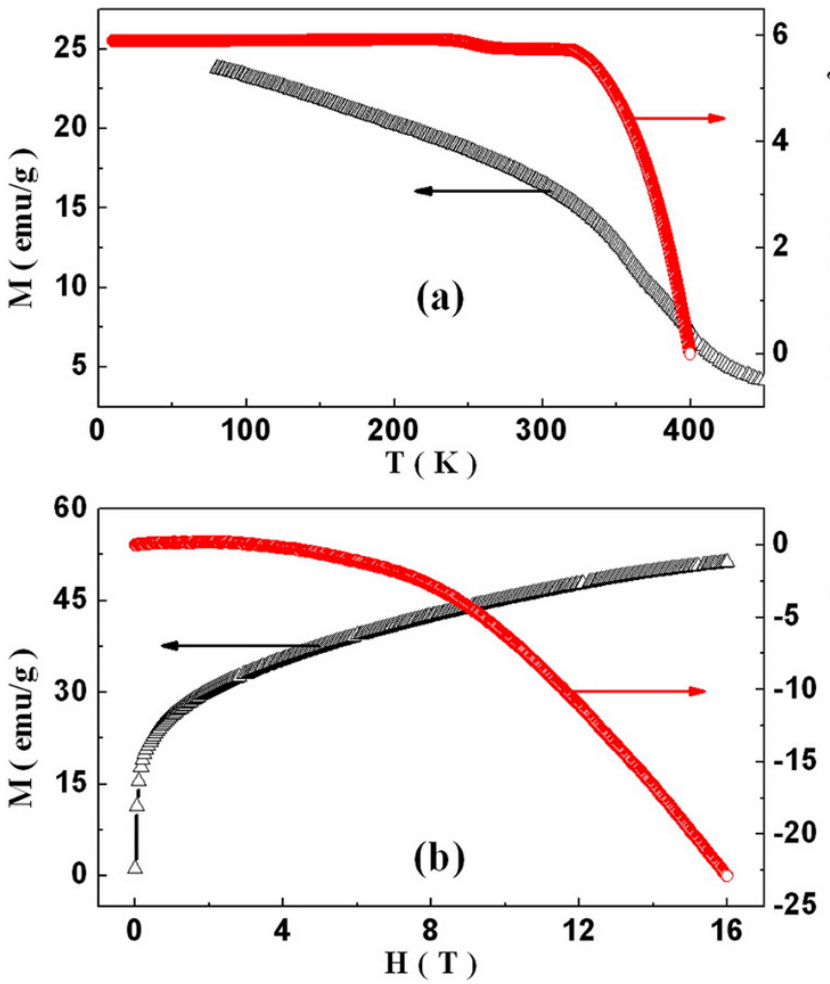

Figure $3 \mid$ (a) The temperature dependence of $P$ (red) and $M$ (black) for SCTFO. (b) The magnetic initial curve (black) and $H$ dependence of $P$ (red) at $300 \mathrm{~K}$.

Comparing with magnetic field, it is easier to generate an electric field in devices and working with a voltage, rather than an electric current, gives rise to less energy consuming in technological applications $^{5,8,9}$. Therefore, the electric-field controlled ferromagnetism, which is usually defined as converse magnetoelectric effect, is of great importance from the application point of view. However, the investigation of electric control of magnetism has been hampered in many single-phase multiferroics due to their low resistivity ${ }^{8,9,25}$. In the case of SCTFO, the resistivity reaches up to $4.36 \times 10^{10} \Omega . \mathrm{cm}$, which is high enough to support an electric field and ensures the following measurement. We first measured the room-temperature magnetic loops of SCTFO with DC electric field $\left(E_{d c}\right)$ parallel and perpendicular to the $H$, respectively. By applying an $E_{d c}$, an obvious reduction of $M$ is observed and typical loops at zero and $22 \mathrm{kv} / \mathrm{cm}$ field are shown in Fig. 4(a). Similar to the effect of $H$ on $P, M$ decreases with increasing $E_{d c}$ and the change of $M$ with $E_{d c}=22 \mathrm{kv} / \mathrm{cm}$ reaches up to $6.3 \%$ and $2.8 \%$, for $E_{d c} \perp H$ and $E_{d c} \| H$, respectively, as shown in Fig. 4(b).

The variation of $M$ of SCTFO as a function of $E_{d c}$ was measured without magnetic bias field. Before this measurement, the sample was pre-magnetized in a field of $5 \mathrm{kOe}$. A considerable reduction of $M$ is observed in Fig. 5(a) despite of positive or negative $E_{d c}$. This result is of interest since no $H$ is utilized in electric control of magnetism. Figure 5(b) shows the time dependence of $M$ under a square wave electric field of $18.5 \mathrm{kV} / \mathrm{cm}$. $M$ decreases or increases with applying or removing $E_{d c}$, respectively, indicating a stable response to the electric field, which demonstrates the modulation of $E$ on $M$ in SCTFO.

In order to further investigate the effect of $E$ on $M$, the scanning probe microscopy (SPM) experiment was performed using lift mode. Figure 6(a) is the topography of a selected area, while Fig. 6(b)-6(d) show the magnetic force microscopy (MFM) images for the same scanning area, which reveal magnetic contrast between the surface magnetization domains. During the MFM studies, various electric fields were applied on the sample. In this case, the bias electric field
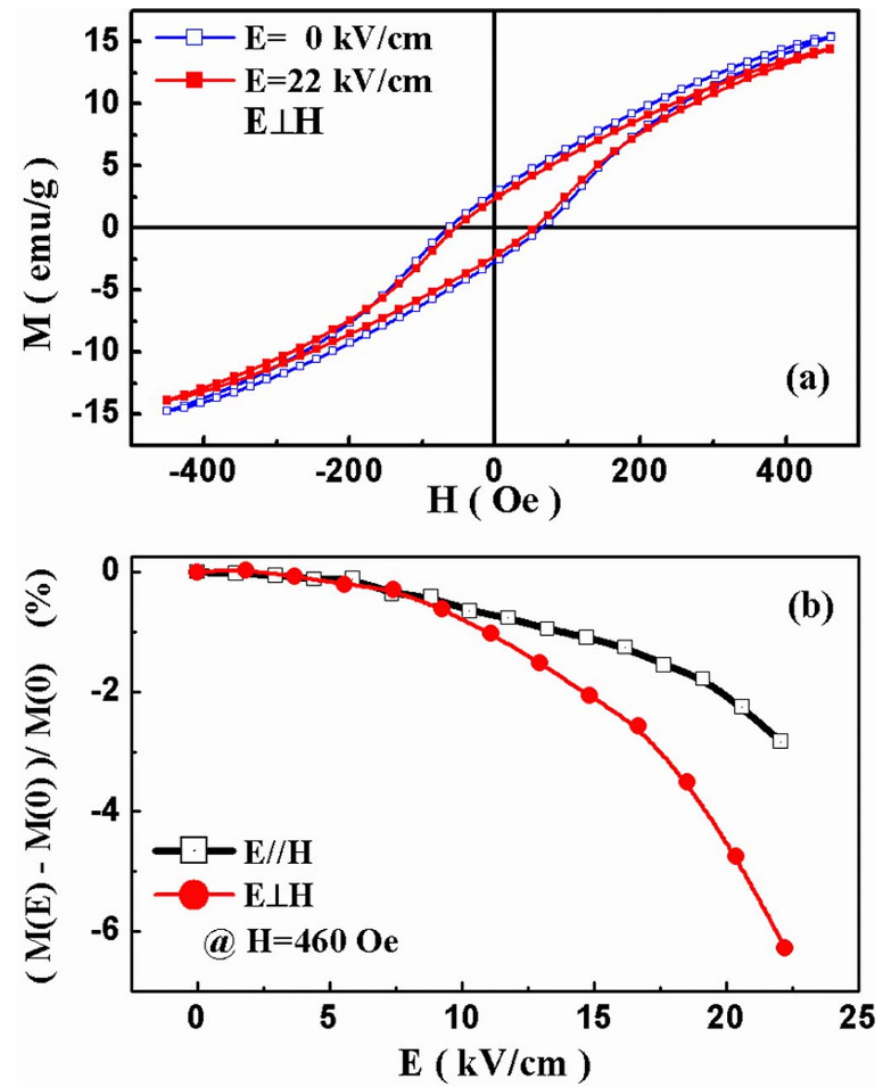

Figure $4 \mid$ (a) The room temperature magnetic hysteresis loop under different $E_{d c}$ (zero and $22 \mathrm{kV} / \mathrm{cm}$ ); (b) The change rate of $M$ under various $E_{d c}$ with magnetic bias field ( $H=460 \mathrm{Oe}$ ) at room temperature: $E \perp H$ (Red); $E \| H$ (Black).
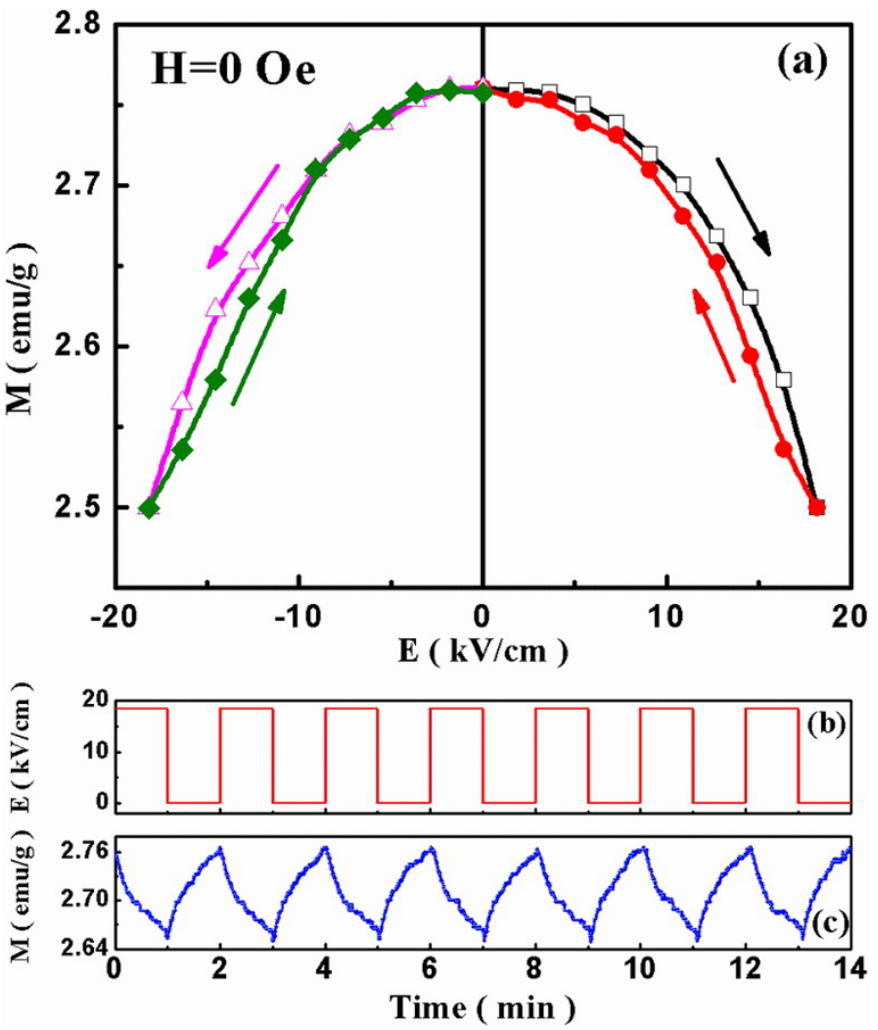

Figure 5 (a) The change of $M$ as a function of $E_{d c}$ at room temperature without magnetic bias field; (b) The time dependence of $M$ under a square wave electric field. 
(a) Topography

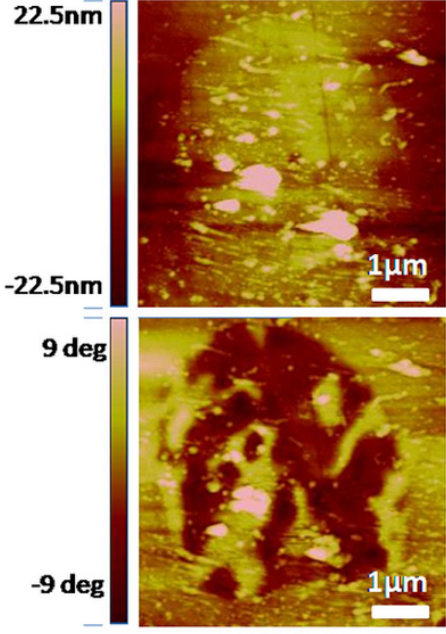

(c) $\mathrm{V}=6 \mathrm{~V}$ (b) $\mathrm{V}=\mathbf{0 V}$

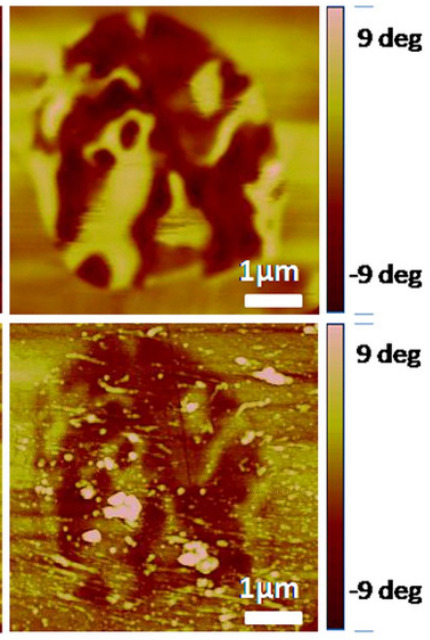

(d) $\mathrm{V}=10 \mathrm{~V}$
Figure 6 | Topography (a) and MFM images of SCTFO at a selected area under different poling voltage: (b) $\mathrm{V}=0 \mathrm{~V}$; (c) $\mathrm{V}=6 \mathrm{~V}$; (d) $\mathrm{V}=10 \mathrm{~V}$.

would make the magnetic force signal shift uniformly, but would not affect the contrast of the signal. From Fig. 6, no change in domain structure can be detected, but the magnetic contrast fades out as electric voltage increasing from 0 to $10 \mathrm{~V}$, indicating the effect of $E$ on the magnetic domain. This experimental result is significant since it gives a direct and powerful evidence of electric control of magnetism at room temperature.

\section{Discussion}

In the single-phase SCTFO, the doping of $\mathrm{Co}^{2+}$ is propitious to change the magnetic anisotropy from axial to planar, while the substitution of $\mathrm{Ti}^{4+}$ interrupts the magnetic interactions between the two blocks RS and $\mathrm{R}^{*} \mathrm{~S}^{* 15,20,21}$. As a result, the magnetic frustration at the boundary stabilizes the nonlinear spiral spin structure, inducing the spontaneous ferroelectric polarization at room temperature, which is attributed to the spin current or the inverse Dzyaloshinskii-Moriya models. In present sample, the substitution of a $\mathrm{Co}^{2+}-\mathrm{Ti}^{4+}$ pair can maintain the average $3+$ valence state and then suppress the hopping of electrons between $\mathrm{Fe}^{2+}$ and $\mathrm{Fe}^{3+}$ in SCTFO. In addition, sintering in oxygen is also helpful to increase the resistivity. As a result, SCTFO has a high enough resistivity which ensures the magnetoelectric measurements. As shown in Fig. 3(b), the values of P decrease monotonously with the increasing magnetic field. According to Eq. (1), the spin cone in SCTFO tends to close with a magnetic field, leading to the decrease of $P$ correspondingly ${ }^{14}$. As for the effect of $E$ on $M$, the values of $M$ decreases with increasing $E$, which is obviously shown in Fig. 4, 5, and 6. When an electric field is applied on the sample, the electric polarization would intrinsically increase. According to Eq. 1, the value of $\bar{S}_{i} \times \bar{S}_{j}$ would increase and lead to the enlargement of the spin cone angle correspondingly, which results in the reduction of magnetization in our sample. Since the electric control of magnetism is of significance, the effect of $\mathrm{E}$ on $\mathrm{M}$ without magnetic bias field is emphasized in present work. As shown in Fig. 5, the remanent magnetization of SCTFO can be reversibly and reproducibly controlled by an electric field, suggesting the potential application in the nonvolatileswelling RAM with multiferroic hexaferrites.

Thus we have prepared a single-phase multiferroics SCTFO. The substitution of $\mathrm{Co}^{2+}-\mathrm{Ti}^{4+}$ pair for two $\mathrm{Fe}^{3+}$ cations produces a spiral conical magnetic structure and high resistivity in SCTFO. This special spin structure can induce spontaneous $P$ via spin-orbit coupling, suggesting the strong magnetoelectric coupling in SCTFO. Electric control of magnetism is confirmed by magnetization and MFM measurements. The excellent magnetoelectric performance in SCTFO gives a valuable contribution to the study of magnetoelectric effect and enables the realization of electric control magnetization in magnetoelectric devices.

\section{Methods}

Polycrystalline sample of $\mathrm{SrCo}_{2} \mathrm{Ti}_{2} \mathrm{Fe}_{8} \mathrm{O}_{19}$ was prepared through conventional solidstate reaction. The high-purity powders of $\mathrm{SrCO}_{3}, \mathrm{Fe}_{2} \mathrm{O}_{3}, \mathrm{Co}_{2} \mathrm{O}_{3}$, and $\mathrm{TiO}_{2}$ were mixed stoichiometrically and sintered at $1,200^{\circ} \mathrm{C}$ for 10 hours for twice in the oxygen atmosphere. The powder X-ray diffraction measurement was carried out to identify the sample as single phase. For the electric polarization measurements, a specimen was cut with a dimension of $3 \times 3 \times 1 \mathrm{~mm}^{3}$ and gold electrodes were painted on both sides as a capacitance. The temperature or magnetic field dependence of $\mathrm{P}$ was obtained by integrating the magnetoelectric current, where temperature and applied $H$ were controlled by the physical property measurement system (PPMS, Quantum Design) and magnetoelectric current was gathered by an electrometer (model 6514, Keithley). The magnetic properties of $\mathrm{SrCo}_{2} \mathrm{Ti}_{2} \mathrm{Fe}_{8} \mathrm{O}_{19}$ were measured with a commercial vibrating sample magnetometer (VSM, model 7407, Lakeshore). The effect of $E_{d c}$ on $M$ was measured with a VSM under zero magnetic field at room temperature. During measurement, $E_{d c}$ was applied on the capacitance and the induced $M$ was detected by pick up coils. The MFM measurements were carried out on the polished sample by scanning probe microscopy (SPM) (Dimension V, Veeco). In order to investigate the effect of $E$ on the magnetic domain, various voltages were applied between the probe tip and the sample chuck. During the MFM measuring processes, two different modes, "grounding the tip" and "applying the voltage on the tip", are used.

1. Fiebig, M. Revival of the magnetoelectric effect. J. Phys. D. Appl. Phys. 38, R123R152 (2005).

2. Eerenstein, W., Mathur, N. D. \& Scott, J. F. Multiferroic and magnetoelectric materials. Nature 442,759-765 (2006)

3. Tokura, Y. Multiferroics as Quantum Electromagnets. Science 312, 1481-1482 (2006).

4. Ramesh, R. \& Spaldin, N. A. Multiferroics: progress and prospects in thin films. Nat. Mater 6, 21-29 (2007).

5. Zhao, T. et al. Electrical control of antiferromagnetic domains in multiferroic $\mathrm{BiFeO}_{3}$ films at room temperature. Nat. Mater. 5, 823-829 (2006).

6. Hur, N. et al. Electric polarization reversal and memory in a multiferroic material induced by magnetic fields. Nature 429, 392-395 (2004).

7. Kimura, T. et al. Magnetic control of ferroelectric polarization. Nature 426, 55-58 (2003).

8. Fennie, C. J. \& Schlom, D. G. Resistance is not futile. Nat. Mater. 9, 787-788 (2010).

9. Bibes, M. \& Barthélémy, A. Multiferroics: Towards a magnetoelectric memory. Nat. Mater. 7, 425-426 (2008).

10. Kimura, T., Lawes, G., \& Ramirez, A. P. Electric Polarization Rotation in a Hexaferrite with Long-Wavelength Magnetic Structures. Phys. Rev. Lett. 94, 137201 (2005).

11. Ishiwata, S., Taguchi, Y., Murakawa, H., Onose, Y., \& Tokura Y. Low-MagneticField Control of Electric Polarization Vector in a Helimagnet. Science 319, 1643 1646 (2008).

12. Chun, S. H. et al. Realization of Giant Magnetoelectricity in Helimagnets. Phys. Rev. Lett. 104, 037204 (2010).

13. Kitagawa, Y. et al. Low-field magnetoelectric effect at room temperature. Nat. Mater. 9, 797-802 (2010).

14. Tokunaga, Y. et al. Multiferroic $M$-Type Hexaferrites with a Room-Temperature Conical State and Magnetically Controllable Spin Helicity. Phys. Rev. Lett. 105, 257201 (2010).

15. Özgür, Ü., Alivov, Y., \& Morkoç, H. Microwave ferrites, part 1: fundamental properties. J. Mater. Sci.-Mater. Electron. 20, 789-834 (2009).

16. Kimura, T. Spiral Magnets as Magnetoelectrics. Annu. Rev. Mater. Res. 37, 387413 (2007).

17. Khomskii, D. Classifying multiferroics: Mechanisms and effects. Physics 2, 20 (2009).

18. Katsura, H., Nagaosa, N., \& Balatsky, A. V. Spin Current and Magnetoelectric Effect in Noncollinear Magnets. Phys. Rev. Lett. 95, 057205 (2005).

19. Koga, N. \& Tsutaoka, T. Preparation of substituted barium ferrite $\mathrm{BaFe}_{12-\mathrm{x}}\left(\mathrm{Ti}_{0.5} \mathrm{Co}_{0.5}\right)_{\mathrm{x}} \mathrm{O}_{19}$ by citrate precursor method and compositional dependence of their magnetic properties. J. Magn. Magn. Mater. 313, 168-175 (2007).

20. Kreisel, J., Vincent, H., Tasset, F., Paté, M., \& Ganne, J. P. An investigation of the magnetic anisotropy change in $\mathrm{BaFe}_{12-2 \mathrm{x}} \mathrm{Ti}_{\mathrm{x}} \mathrm{Co}_{\mathrm{x}} \mathrm{O}_{19}$ single crystals. J. Magn. Magn. Mater. 224, 17-29 (2001).

21. Batlle, X. et al. Cation distribution and intrinsic magnetic properties of Co-Ti doped M-type ferrite. J. Appl. Phys. 70, 1614-1623 (1991).

22. Williams, J. M., Adetunji, J., \& Gregori, M. Mössbauer spectroscopic determination of magnetic moments of $\mathrm{Fe}^{3+}$ and $\mathrm{Co}^{2+}$ in substituted barium hexaferrite, $\mathrm{Ba}(\mathrm{Co}, \mathrm{Ti})_{\mathrm{x}} \mathrm{Fe}_{(12-2 \mathrm{x})} \mathrm{O}_{19}$. J. Magn. Magn. Mater. 220, 124-128 (2000). 
23. Wohlfarth, E. P. Handbook of Magnetic Materials. Volume 3, 325-328 (NorthHolland Publishing Company, 1982).

24. Teh, G. B., Saravanan, N., \& Jefferson, D. A. A study of magnetoplumbite-type (Mtype) cobalt-titanium-substituted barium ferrite, $\mathrm{BaCo}_{\mathrm{x}} \mathrm{Ti}_{\mathrm{x}} \mathrm{Fe}_{12-2 \mathrm{x}} \mathrm{O}_{19}(\mathrm{x}=1-6)$. Mater. Chem. Phys. 105, 253-259 (2007).

25. Lee, J. H. et al. A strong ferroelectric ferromagnet created by means of spin-lattice coupling. Nature 466, 954-959 (2010).

\section{Acknowledgement}

We acknowledge Zhengcai Xia for his support in measurements. This work is supported by the National Basic Research Program of China (2009CB929501 and 2012CB932304),

National Natural Science Foundation of China (Grant No. 50971069 and 11174130).

\section{Author contribution}

L.Y.W, prepared the sample; L.Y.W and D.H.W, designed the experiments and wrote the paper. L.Y.W., H.C.X. and J.L.G. performed the measurements. L.Y.W., D.H.W., Q.Q.C., and Y.X.Z. collected and analyzed the data. All authors discussed the results and commented on the manuscript.

\section{Additional information}

Competing financial interests: The authors declare no competing financial interests.

License: This work is licensed under a Creative Commons

Attribution-NonCommercial-NoDerivative Works 3.0 Unported License. To view a copy of this license, visit http://creativecommons.org/licenses/by-nc-nd/3.0/

How to cite this article: Wang, L. et al. Electric control of magnetism at room temperature. Sci. Rep. 2, 223; DOI:10.1038/srep00223 (2012). 